\title{
Robust Imaging Methodology for Challenging Environments: Wave Equation Dispersion Inversion of Surface Waves Jing Li ${ }^{1,2}$, Gerard Schuster ${ }^{l}$ and Zhaofa Zeng ${ }^{2}$ \\ ${ }^{1}$ King Abdullah University of Science and Technology (KAUST), Saudi Arabia \\ ${ }^{2}$ Collage of geo-exploration Sci. \&Tech, Jilin University, Changchun, Jilin, China
}

\section{Summary}

A robust imaging technology is reviewed that provide subsurface information in challenging environments: waveequation dispersion inversion (WD) of surface waves for the shear velocity model. We demonstrate the benefits and liabilities of the method with synthetic seismograms and field data. The benefits of WD are that 1) there is no layered medium assumption, as there is in conventional inversion of dispersion curves, so that the $2 \mathrm{D}$ or $3 \mathrm{D}$ Svelocity model can be reliably obtained with seismic surveys over rugged topography, and 2) WD mostly avoids getting stuck in local minima. The synthetic and field data examples demonstrate that WD can accurately reconstruct the S-wave velocity distributions in laterally heterogeneous media if the dispersion curves can be identified and picked. The WD method is easily extended to anisotropic media and the inversion of dispersion curves associated with Love wave. The liability is that is almost as expensive as FWI and only recovers the Vs distribution to a depth no deeper than about $1 / 2 \sim 1 / 3$ wavelength.

\section{Introduction}

Inverting surface waves for the S-wave velocity model falls into two categories: 1) the classical method of inverting dispersion curves (Evison et al., 1959; Park et al., 1998) for a 1D layered medium, and 2) full waveform inversion (FWI) (Groos et al., 2014; Solano et al., 2014; Yuan et al., 2015) for 2D and 3D media. The classical method accurately inverts for a 1D S-wave velocity model, but becomes less accurate with increasing lateral heterogeneity in the subsurface. The 1D assumption is not satisfied for some practical applications, so partial remedies are spatial interpolation of 1D velocity models (Xia et al., 1999) and laterally constrained inversion (Socco et al., 2010; Bergamo et al., 2012). In comparison, full waveform inversion (FWI) can theoretically account for any lateral heterogeneity, but it is computationally expensive and can easily get stuck in local minima associated with the objective function (Tarantola, 1984).

To avoid falling into a local minimum, the initial model should be smooth and time-damping strategies can be used at the early iterations (Sheng et al., 2006; Brossier et al., 2009; Romdhane et al., 2011). However, there are no failsafe strategies for always avoiding local minima in the context of FWI with surface waves. A partial FWI method is that of Perez Solano et al. (2014) who used the magnitude spectra of surface waves as the input data. Results with some synthetic data showed this to be a robust and efficient method for reconstructing the S-wave velocity model at the near surface. Another surface-wave inversion strategy is proposed by Yuan et al. (2015), who developed a wavelet multi-scale adjoint method for the joint inversion of both surface and body waves. Synthetic tests showed that this approach can avoid cycle skipping for some models. The role of attenuation in FWI with surface waves was studied by Groos et al. (2014). They concluded that the estimation of a priori quality factors is critical for inverting seismic waves in the near-surface zone. Instead of inverting Rayleigh waves.

\section{Wave Equation Dispersion Inversion}

Wave-equation dispersion inversion (WD) of surface waves is mostly restricted to inverting the dispersion curve associated with Rayleigh waves for 1D Vs model (Aki and Richards, 1980). This method has been used successfully for estimating shallow 1D Vs models from controlled source data as well as for imaging crustal models from teleseismic data. The WD procedure is more robust than FWI because it replaces complicated surface-wave arrivals with simple dispersion curves in the wavenumber $k_{x}-\omega$ or phase-velocity $C(\omega)-\omega$ domains in Figure 1. The WD method presented in this paper is the adjoint-state method presented by ( $\mathrm{Li}$ et al., 2016), who used a difference approximation to the gradient rather than an adjoint operation. Hence, our WD method is more than an order-ofmagnitude faster for complicated models.

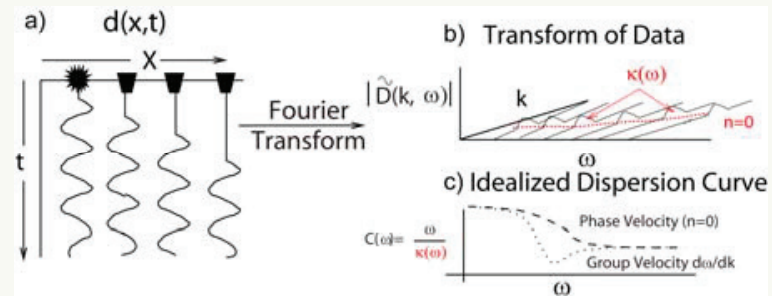

Figure 1: a) Common shot gather $\mathrm{d}(\mathrm{x}, \mathrm{t}), \mathrm{b})$ the fundamental $(\mathrm{n}=0)$ dispersion curves for Rayleigh waves in the $k_{x}-\omega$ domain, and c) $C(\omega)-\omega$ domains. Here the phase velocity is $C(\omega)=\omega / k(\omega)$ and $k(\omega)$ is the skeletonized data (Zhang et al., 2015). 


\section{Wave Equation Dispersion Inversion}

\section{Theory and Practice}

We will introduce the theories and workflows for WD. Each description will be accompanied by results using both synthetic seismograms and field data. The mathematical details for WD theory are presented in Li et al. (2017), and the workflow for WD is described below and in Figure 1.

1. Radon and FFT transforms applied to CSGs: The fundamental-mode Rayleigh waves are windowed in a common shot gather (CSG), and an FFT and Radon transform are applied to get the spectrum $D(c, \omega)$, where $c$ is the phase velocity and $\omega$ is frequency. From this spectra the fundamental dispersion curve $C(\omega)$ is picked to get $\kappa(\omega)=\omega / C(\omega)$. We use the superscript obs if the data are recorded, otherwise it is predicted data computed by a finite-difference solution to the $2 \mathrm{D}$ elastic wave equation.

1. The misfit function is computed to get

$$
\varepsilon=\frac{1}{2} \sum_{\omega}\left(\kappa(\omega)-\kappa(\omega)^{o b s}\right)^{2},
$$

where an extra summation is over shot indexes if there is more than one shot gather.

2. The gradient with respect to the shear-slowness model $\mathrm{s}(\mathbf{x})$ is computed to get

$$
\gamma(x)=\frac{\partial \varepsilon}{\partial s(x)}=\sum_{\omega} \Delta \kappa(\omega) \frac{\partial \kappa}{\partial s(x)}
$$

and the steepest descent formula is

$$
s(x)^{k+1}=s(x)^{k}-\alpha \sum_{\omega} \Delta \kappa(\omega) \frac{\partial \kappa}{\partial s(x)},
$$

where the Fre chet derivative is computed using solutions to the $2 \mathrm{D}$ elastic wave equation. If there is more than one shot gather then there is an extra summation over different indices in equation 3 .

3. Equation 3 is used (typically, no more 10-15 iterations) to get the shear-velocity slowness model $s(x)^{k}$ until the data residual falls below some accepted criterion. This methodology is valid for both $2 \mathrm{D}$ and $3 \mathrm{D}$ velocity models and eliminates the layered medium assumption in traditional dispersion inversion methods. Figure 2 depicts the workflow for the surface-wave WD inversion.

a) Use window muting to remove the first-arrival body waves, backscattered data and higher modes of the Rayleigh waves in the shot gather and then apply a 1D Fourier transform along the $x_{g}$ coordinate of the shot gather to get its spectrum. The same muting is applied to both the observed data and the predicted shot gather computed by a finite- difference solution to the elastic wave equation.

b) Apply a linear Radon transform (LRT) to the spectra of predicted and observed data to get the phasevelocity image in the $\omega-C$ domain, where $\mathrm{C}$ is the phase velocity of the surface waves. The fundamental dispersion-curves are automatically picked according to the maximum amplitudes of the magnitude spectrum. However, in some field data, the dispersion curves still contain higher-order modes, so there will be discontinuities in the dispersion curves.

c) Calculate the weighted data $\hat{D}(g, \omega)_{o b s}$, which can be used to compute the backprojected data ( $\mathrm{Li}$, et al, 2017). The forward propagated source has the weighted source field.

d) Estimate the step-length $\alpha$ by any backtracking linesearch method (Nocedal, and Wright, 1999).

e) The gradients for each migrated shot gather are added together to get the S-wave velocity update (equation 3 ). The background S-wave velocity model is updated and the above steps are repeated until the residual falls below a specified value.

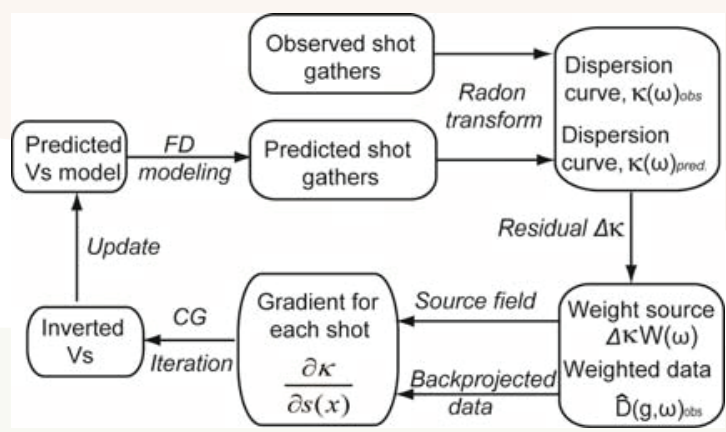

Figure 2. Workflow for the WD method.

\section{WD Numerical Results}

The WD method is tested on the S-velocity model shown in Figure 3a, where the starting velocity model for WD is depicted in Figure $3 \mathrm{~b}$. The input data were 100 shot gathers ( $2 \mathrm{~m}$ shot intervals) with 100 traces $(2 \mathrm{~m}$ spacing) per shot gather, and each shot gather was transformed into the k- $\omega$ domain in order to pick the fundamental dispersion curve $\kappa(\omega)^{o b s}$. Finite-difference solutions to the 2D elastic wave equation are computed to get the predicted dispersion values $\kappa(\omega)$, and each dispersion curve is inverted separately to get a $1 \mathrm{D}$ velocity profile. These $1 \mathrm{D}$ velocity 


\section{Wave Equation Dispersion Inversion}

profiles are assembled to give the 1D WD tomogram in Figure $3 \mathrm{~d}$, which has significant distortion in the shapes and locations of the velocity anomalies. Inverting all of these dispersion curves simultaneously (without assuming a 1D model) gives the 2D S-velocity tomogram in Figure 3c. It is obvious that this tomogram is an accurate rendering of a smoothed version of the actual model in Figure 3a.
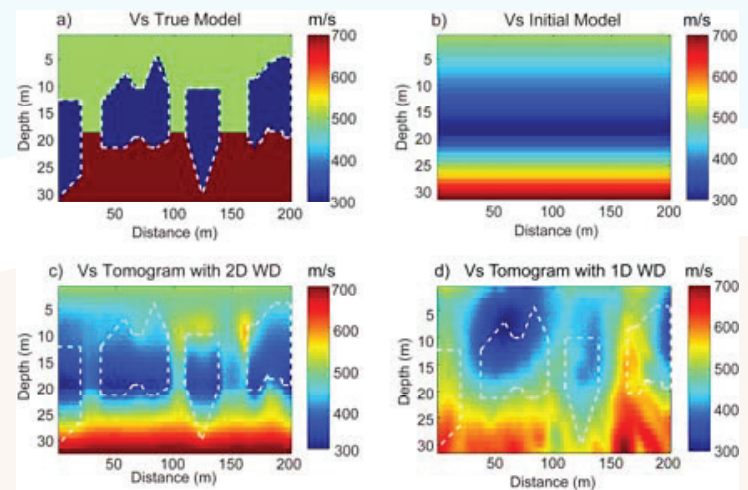

Figure 3. a) Actual and b) starting S-velocity models, c) 2D WD tomogram, and d) 1D WD tomogram that assumes a 1D layered model beneath each shot point (Figures from $\mathrm{Li}$ et al. (2017)).

\section{Complex Foothill Model Test}
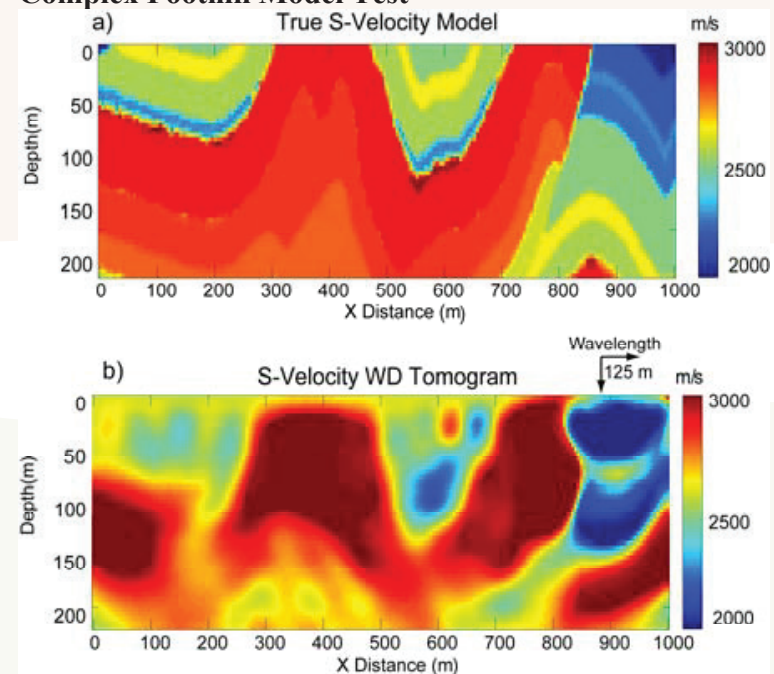

Figure 4: a) The Foothill S-velocity model and b) WD Svelocity tomogram.

Foothills regions are recognized as some of the most challenging areas for onshore seismic exploration. Complex geology at the near surface introduces serious problems for static corrections and the signal-to-noise ratio. Foothill velocity model shown in Figure 8a is a portion of the model described by Gray (1998) depicts a number of faults and folded layers (typical of Foothills mountainous thrust regions). Seismic data are computed for the shot and receiver spacing of $20 \mathrm{~m}$ on the free surface, and there are 50 traces per shot gather to give a total of 50 shot gathers. The goals are to estimate the basin depth as well as the near-surface geology, where the source function is a Ricker wavelet peaked at $10 \mathrm{~Hz}$. The dispersion curves are picked and inverted. The resulting S-wave velocity tomogram is shown in Figure $4 \mathrm{~b}$. Here, the reconstructed fault structures are consistent with the faults in the true model (Figure $4 \mathrm{a}$ ).

\section{Field Data Test}

The field data test is carried out on seismic data recorded over a basin near Olduvai Gorge, Tanzania which is one of the earliest hominid sites where fossil and stone tool evidence dates back to more than 2.2 million years. A 2D seismic survey with a total length of $3.6 \mathrm{~km}$ is recorded and the survey line straddles the major Fifth Fault. The survey line consists of 3 profiles, each of which has a length of $1.2 \mathrm{~km}$, with 240 channels at $5 \mathrm{~m}$ receiver intervals. In-line shooting is carried out for all 3 profiles, with 120 shots at $10 \mathrm{~m}$ intervals for each profile. We use a $200 \mathrm{lb}$ accelerated weight drop source, and stack 30 shot records at each shot location.

We can pick the surface-wave fundamental mode dispersion curves. Simultaneously inverting all of the dispersion curves by the 2D WD method gives the S-wave velocity tomogram shown in Figure 5a, where the dashed lines are interpreted as fault structures. The Fifth Fault and other faults are indicated in the S-wave velocity tomogram. This diagnosis is consistent with the interpretation of faults by the P-wave velocity tomogram in Figure $5 \mathrm{~b}$ and the common offset gather (COG) profile in Figure $5 \mathrm{c}$. In addition, the S-wave tomogram computed by the WD method provides a more detailed velocity structure in the shallow regions. Here, a fault is suggested by a sudden delay in the onset of surface waves at the dashed lines. The combination of both the S-wave and P-wave velocity tomograms and the COG strongly suggest the presence of fault like structures which gives a new interpretation to the geology in this basin.

\section{Conclusions}

Seismic data recorded in rugged foothill environments require rugged imaging methods that will be up to the challenge of low SNR and rugged topography. The surface waves are always the strongest arrivals so their inversion by WD will yield the most robust results. WD easily accommodates rugged topography because the surface wave data can be predicted by FD solutions for sources and receivers on a $3 \mathrm{D}$ topographic surface. The S-velocity tomogram can be used to estimate $\mathrm{S}$-wave statics for multicomponent data and assess locations of near-surface faults for drilling hazard assessment. 


\section{Wave Equation Dispersion Inversion}
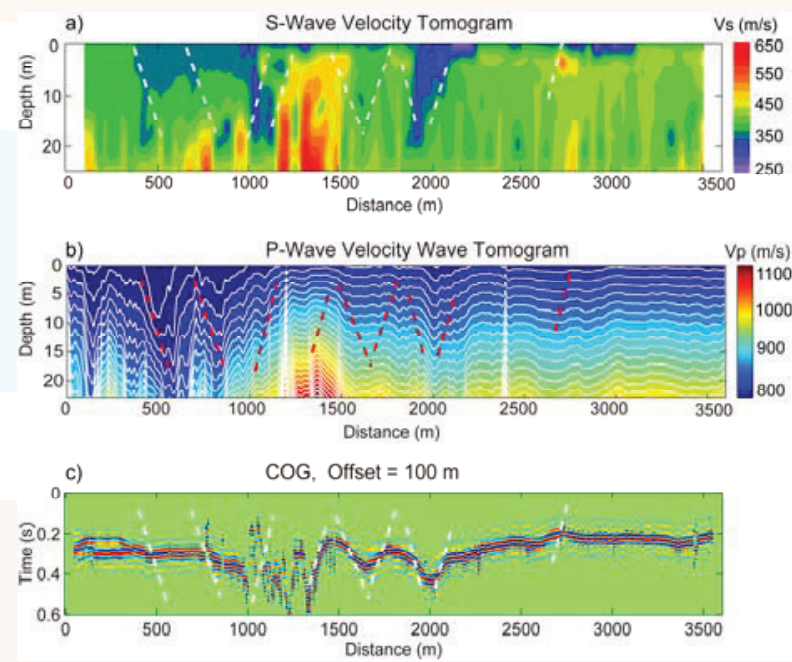

Figure 5. a) S-wave velocity tomogram obtained by inverting the dispersion curves computed from the East Africa data, b) P-wave velocity tomogram by wave equation traveltime inversion of first arrivals, and c) COGs at the source-receiver offset of $100 \mathrm{~m}$ for data recorded by an East Africa survey. The dashed white faults are more reliably interpreted than the dashed red faults (Figures from Li et al. (2017)).

\section{Acknowledgements}

We thank the sponsors of the CSIM consortium for their financial support. We also thank the KAUST supercomputing center for use of their supercomputer.

\section{References}

Aki, K., and P. Richards, 1980, Quantitative seismology: Theory and methods: San Francisco.

Bergamo, P., D. Boiero, and L. V. Socco, 2012, Retrieving 2D structures from surface-wave data by means of spacevarying spatial windowing: Geophysics, 77, EN39-EN51.

Brossier, R., S. Operto, and J. Virieux, 2009, Seismic imaging of complex onshore structures by $2 \mathrm{~d}$ elastic frequency-domain full-waveform inversion: Geophysics, 74, WCC105-WCC118.

Evison, F. F., R. Orr, and C. Ingham, 1959, Thickness of the earth as crust in antarctica: Nature, 306-308.

Groos, L., M. Sch“afer, T. Forbriger, and T. Bohlen, 2014, The role of attenuation in $2 \mathrm{~d}$ full-waveform inversion of shallow-seismic body and rayleigh waves: Geophysics, 79, R247-R261.
Gray, S. H., 1998, Interpretive seismic imaging in structurally complex areas: 282-285, CSPG Special Publications.

Lu, K, J Li, B Guo, L Fu, G Schuster, 2017, Tutorial for wave-equation inversion of skeletonized data: Interpretation 5 (3), SO1-SO10.

Li, J., and G. Schuster, 2016a, Skeletonized wave equation of surface wave dispersion inversion: 86th Annual International Meeting, SEG, Expanded Abstracts, 36303635.

Li, J., Z. C. Feng, and G. T. Schuster, 2017, Wave-equation dispersion inversion: Geophysical Journal International 208 (3), 1567-1578.

Nocedal, J., and S.Wright, 2006, Numerical optimization: Springer Science \& Business Media.

Park, C. B., R. D. Miller, J. Xia, et al., 1998, Imaging dispersion curves of surface waves on multi-channel record: SEG Expanded Abstracts, 1377-1380.

Romdhane, A., G. Grandjean, R. Brossier, F. R'ejiba, S. Operto, and J. Virieux, 2011, Shallow-structure characterization by $2 \mathrm{~d}$ elastic full-waveform inversion: Geophysics, 76, R81-R93.

Sheng, J., A. Leeds, M. Buddensiek, and G. T. Schuster, 2006, Early arrival waveform tomography on near-surface refraction data: Geophysics, 71, U47-U57.

Socco, L. V., S. Foti, and D. Boiero, 2010, Surface wave analysis for building near-surface velocity models established approaches and new perspectives: Geophysics, 75, 75A $83-75 \mathrm{~A} 102$.

Solano, C. P., D. Donno, and H. Chauris, 2014, Alternative waveform inversion for surface wave analysis in 2-d media: Geophysical Journal International, 198, 1359-1372.

Tarantola, A., 1984, Inversion of seismic reflection data in the acoustic approximation: Geophysics, 49, 1259-1266.

Xia, J., R. D. Miller, and C. B. Park, 1999, Estiation of near-surface shear-wave veloeity by inversion of Rayleigh wave: Geophysics, 691-700.

Yuan, Y. O., F. J. Simons, and E. Bozdag, 2015, Multiscale adjoint waveform tomography for surface and body waves: Geophysics, 80, R281-R302.

Zhang, Z., Y. Liu, and G. Schuster, 2015, Wave equation inversion of skeletonized surface waves: SEG Technical Program Expanded Abstracts, 2391-2395. 